\title{
Effect of high-temperature heat treatment duration on the purity and microstructure of MWCNTs
}

\author{
I SRIKANTH ${ }^{1,2, *}$, N PADMAVATHI ${ }^{2}$, P S R PRASAD ${ }^{3}$, P GHOSAL $^{4}$, R K JAIN $^{1}$ and \\ CH SUBRAHMANYAM ${ }^{1}$ \\ ${ }^{1}$ Indian Institute of Technology, Hyderabad 502 205, India \\ ${ }^{2}$ Advanced Systems Laboratory, DRDO, Hyderabad 500 058, India \\ ${ }^{3}$ National Geophysical Research Institute (CSIR-NGRI), Hyderabad, India \\ ${ }^{4}$ Defence Metallurgical Research Laboratory, DRDO, Hyderabad 500 058, India
}

MS received 4 June 2014; accepted 27 October 2014

\begin{abstract}
The effect of high-temperature heat treatment on purity and structural changes of multiwalled carbon nanotubes (MWCNTs) were studied by subjecting the raw MWCNTs (pristine MWCNTs) to $2600^{\circ} \mathrm{C}$ for 60 and 120 min. Thermogravimetric analysis (TGA), X-ray diffraction, Raman spectroscopy, transmission electron microscopy (TEM) and scanning electron microscopy (SEM) were used to study the effect of heat-treatment duration on the purity and structural changes of MWCNTs. Results show that high-temperature heat treatment can be used to purify MWCNTs with proper optimization of treatment time. It was observed that 60 min heat treatment of raw MWCNTs imparts high purity and structural perfection to MWCNTs, while $120 \mathrm{~min}$ heat treatment imparts structural degradation to MWCNTs with collapse of the innermost shells. The present study indicates that metal impurities act as moderators in controlling the degradation of MWCNTs up to certain duration, and once the metal impurities escape completely, further heat treatment degrades the structure of MWCNTs.
\end{abstract}

Keywords. Carbon nanotubes; graphitization; X-ray diffraction; electron microscopy.

\section{Introduction}

Large-scale synthesis of multiwalled carbon nanotubes (MWCNTs) is generally carried out by chemical vapour deposition (CVD) process. This method involves decomposition of hydrocarbons at a prescribed temperature and pressure under controlled quantities of catalysts [1]. In general, either transition metals (d-block elements like iron, nickel, etc.) or organometallic complexes (e.g., ferrocene) are decomposed to generate the metal catalyst. Although catalyst helps in the generation of carbon nanotubes (CNTs) with controlled diameter, it remains in the final product as an impurity. As a result of this as-synthesized MWCNTs contain metal catalysts as impurities. Besides the metal catalysts, MWCNTs are also known to contain other carbonaceous impurities such as amorphous carbon. Removal of impurities is the first essential step to use CNTs for any application.

In general, purification of MWCNTs is carried out by a two-step process [2]. In the first step selective oxidation is carried out to remove the amorphous carbon. This involves subjecting the raw MWCNTs (containing metal impurities along with the amorphous carbon) to an approximate temperature of $450^{\circ} \mathrm{C}$ in air. During this process, amorphous carbon will be selectively oxidized and removed. This is because amorphous carbon is more reactive as compared with

\footnotetext{
*Author for correspondence (i_srikanth@yahoo.co.uk)
}

MWCNTs. In the second step, MWCNTs having metallic impurities are refluxed in strong acids. During this process, metallic impurities are removed by dissolution in strong acids. However, the above method of purification, involving refluxing the MWCNTs in strong acids, suffers from the major drawback of creating defects on MWCNTs as exposing to strong acids is known to cause structural degradation to MWCNTs [3,4].

To overcome the problem of structural damage to the CNTs by the above purification method, alternate purification methods were tried by different research groups. Some of them are magnetic method, centrifugation method, hightemperature heat treatment method, etc. [2]. These methods rely on the differences in the physical size, gravity, magnetic properties or thermal stability of the MWCNTs as compared with the impurities. As these methods do not involve oxidative treatments and acid exposure of MWCNTs for purification, they do not impart any structural damage to the MWCNTs. However, most of these methods are less effective in terms of final degree of purity of MWCNTs. Various purification methods reported for MWCNTs along with their advantages and limitations were reviewed by Peng et al [2] and Sato et al [5]. Among various purification methods that they have summarized, high-temperature heat treatment (above $2500^{\circ} \mathrm{C}$ ) in inert atmosphere is identified as one of the best methods, especially for the purification of large quantities of MWCNTs. This is because, besides 
giving highly pure MWCNTs, this method is also known to give enhanced structural perfection to MWCNTs [6-10].

Although it is widely reported that high-temperature heat treatment gives improved structural perfection to MWCNTs, more understanding on the mechanism involved in the structural changes taking place during the high-temperature treatment is necessary. This is because some unwanted changes to the CNT structure during high-temperature heat treatment like transformation of single-walled carbon nanotubes (SWCNTs) to MWCNTs, disappearance of the inner walls of the MWCNTs and their transformation to doublewalled carbon nanotubes (DWCNTs) were reported [11,12]. On the other hand, formation of carbonaceous material deposit on MWCNTs surfaces at high temperatures was also reported [13]. Table 1 gives the summary of some typical observations reported on the structural changes to CNTs due to high-temperature heat treatment. From table 1, it is clear that many unwanted structural changes take place to CNTs, although CNTs with high purity can be obtained with the high-temperature heat treatment method. Hence, mechanisms involved in high-temperature purifications of CNTs need thorough understanding to obtain CNTs with high purity and also with enhanced structural perfection.

Purification by the high-temperature heat treatment method involves a two-step process. In the first step amorphous carbon and other carbonaceous impurities are removed by heating the MWCNTs at around $450^{\circ} \mathrm{C}$ in air. In the second step, MWCNTs will be heat treated up to approximately $2500^{\circ} \mathrm{C}$ in inert atmosphere to remove all the metallic impurities which will get eliminated by evaporation.

In this work, we have studied the effect of duration (60 and $120 \mathrm{~min}$ ) of high-temperature heat treatment for MWCNTs at $2600^{\circ} \mathrm{C}$ under inert atmosphere on purity and structural changes to MWCNTs.

\section{Raw materials}

MWCNTs synthesized by the chemical vapour deposition method were procured from M/s Chemapal Industries Ltd.,

Table 1. Typical reported microstructural changes to CNTs due to high-temperature heat-treatment method.

\begin{tabular}{|c|c|c|c|c|c|c|}
\hline \multirow[b]{2}{*}{ HTT } & \multirow[b]{2}{*}{ Type of CNTs } & \multicolumn{2}{|c|}{$d$-spacing for $\mathrm{d}_{002}$ (in nanometres) } & \multicolumn{2}{|c|}{$I_{\mathrm{D}} / I_{\mathrm{G}}$ ratio (from Raman) } & \multirow[b]{2}{*}{ Remarks \& reference } \\
\hline & & $\mathrm{P}$ & AA & $\mathrm{P}$ & AA & \\
\hline $2800^{\circ} \mathrm{C} \& 30 \mathrm{mins}$ & MWCNTs & 0.348 & 0.339 & 0.9845 & 0.2315 & $\begin{array}{l}\text { Observed crack like voids, } \\
\text { carbon soot contamination [13] }\end{array}$ \\
\hline $2800^{\circ} \mathrm{C} \& 60 \mathrm{mins}$ & MWCNTs & 0.339 & 0.335 & - & - & $\begin{array}{l}d \text {-spacing changing from base } \\
\text { of the tube to apex of the tube [6] }\end{array}$ \\
\hline $2150^{\circ} \mathrm{C} \& 30 \mathrm{mins}$ & MWCNTs & - & - & 0.9 & 0.45 & $\begin{array}{c}\text { Heating under vacuum can } \\
\text { remove encapsulated metal } \\
\text { impurities even at low } \\
\text { temperatures [7] }\end{array}$ \\
\hline $2500^{\circ} \mathrm{C} \& 300 \mathrm{mins}$ & MWCNTs & - & - & - & - & $\begin{array}{l}\text { Sublimation and disappearance } \\
\text { of inner tubes [11] }\end{array}$ \\
\hline $2250^{\circ} \mathrm{C} \& 45 \mathrm{mins}$ & MWCNTs & 0.3425 & 0.3414 & & & $\begin{array}{c}\text { Increased degree of } \\
\text { graphitization, but microstructural } \\
\text { defects are not completely } \\
\text { removed [8] }\end{array}$ \\
\hline $3000^{\circ} \mathrm{C} \& 45 \mathrm{mins}$ & MWCNTs & 0.3425 & 0.3398 & & & $\begin{array}{l}\text { Microstructural defects are } \\
\text { removed, but side grafts (kinks) } \\
\text { are observed [8] }\end{array}$ \\
\hline $2500^{\circ} \mathrm{C}$ for $30 \mathrm{mins}$ & DWCNTs & - & - & 0.226 & 0.141 & $\begin{array}{l}\text { Disappearance of narrow tubes, } \\
\text { diameter enhancement of inner } \\
\text { tubes, breakage of tubes with } \\
\text { formation of MWCNTs, } \\
\text { flaky carbon [14] }\end{array}$ \\
\hline $2800^{\circ} \mathrm{C} \& 30 \mathrm{mins}$ & DWCNTs & - & - & 0.226 & 0.1306 & $\begin{array}{l}\text { Breakage of DWCNTs, formation } \\
\text { of MWCNTs, flaky carbon [14] }\end{array}$ \\
\hline $2400^{\circ} \mathrm{C} \& 120 \mathrm{mins}$ & SWCNTs & - & - & & & $\begin{array}{l}\text { SWCNTs transformed to } \\
\text { MWCNTs [12] }\end{array}$ \\
\hline $2800^{\circ} \mathrm{C} \& 15 \mathrm{mins}$ & SWCNTs & $\begin{array}{r}\text { Disap } \\
\text { reflec } \\
\text { de } \\
\text { cry }\end{array}$ & $\begin{array}{l}\text { of hk0 } \\
\text { cating } \\
\text { the } \\
\text { der }\end{array}$ & $\begin{array}{l}\text { RBM mod } \\
\text { D-banc }\end{array}$ & $\begin{array}{l}\text { appears, } \\
\text { ts up }\end{array}$ & $\begin{array}{l}\text { Transformation of SWNCTs } \\
\text { to MWCNTs [15] }\end{array}$ \\
\hline
\end{tabular}

HTT: heat treatment time \& temperature, P: prestine, AA: after annealing. 
Mumbai. MWCNTs in the pristine conditions (as procured) were having the following features:

1. Metallic impurities : $36 \mathrm{wt} \%$

2. Diameter : $20-30 \mathrm{~nm}$

3. Length : $2-4 \mu \mathrm{m}$

SEM images of the raw MWCNTs are shown in figure 1. It can be observed that MWCNTs are highly entangled in the as-received form (figure 1a and b). It can be seen that white lustrous pockets of metallic impurities are present along with the raw MWCNTs.

\section{Experimental}

MWCNTs (of approximately $200 \mathrm{~g}$ in a batch) were loaded into a graphite crucible having a volume of $1000 \mathrm{ml}$. Graphite crucible was kept inside the high temperature furnace and heated to $2600^{\circ} \mathrm{C}$ under the nitrogen atmosphere at atmospheric pressure. Typical heating cycle involved heating the furnace up to $1200^{\circ} \mathrm{C}$ at a heating rate of $2^{\circ} \mathrm{C} \mathrm{min}-1$, and then

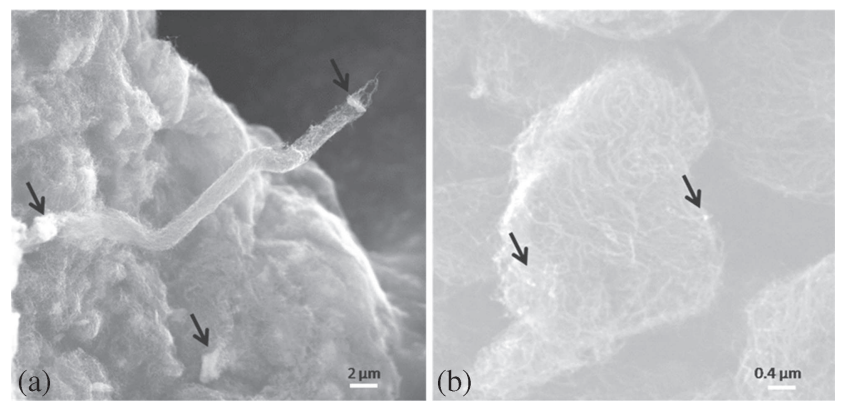

Figure 1. SEM images of the raw MWCNTs: (a) and (b) highly entagled bundles of MWCNTs with white and lustrous metallic impurities (indicated by arrows). raising the temperature from 1200 to $2600^{\circ} \mathrm{C}$ at a heating

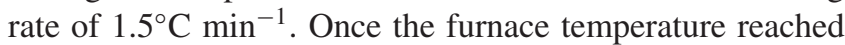
$2600^{\circ} \mathrm{C}$, then soaking (holding the furnace temperature) was carried out. Two different batches of MWCNTs were soaked for different durations namely 60 and $120 \mathrm{~min}$. Hereafter, MWCNTs soaked for $60 \mathrm{~min}$ are referred to as $60 \mathrm{~min}$ heattreated MWCNTs, while the MWCNTs soaked for $120 \mathrm{~min}$ are referred to as 120 min heat-treated MWCNTs. After furnace was cooled to room temperature, MWCNTs, sample was collected and the purity of MWCNTs was measured with thermogravimetric analysis (TGA). TGA was carried out at a heating rate of $10^{\circ} \mathrm{C} \mathrm{min}^{-1}$ from room temperature to $850^{\circ} \mathrm{C}$ under compressed air flow $\left(50 \mathrm{ml} \mathrm{min}^{-1}\right)$. Microstructural changes associated with the heat treatment processes were determined by X-ray diffraction (XRD, Philips PWD, Model No. 1830, The Netherlands), Raman spectroscopy (Jobin Yuan, Model: 64,000X), scanning electron microscopy (ESEM, FEI, Quanta 400, The Netherlands) and transmission electron microscopy (TEM, Tecnai 20, FEI, The Netherlands).

\section{Results and discussion}

Thermogravimetric analysis results of the MWCNTs (raw) and MWCNTs heat treated for 60 min (purified MWCNTs) are shown in figure 2. During TGA studies, in the presence of oxygen and high temperature, CNTs and other carbonaceous impurities burnout leaving behind the metallic impurities. When the carbonaceous materials start to oxidize, it results in a rapid weight loss which can be observed from approximately $550^{\circ} \mathrm{C}$ for the raw MWCNTs. The weight loss continued to increase until it reached a stable plateau region near approximately $650^{\circ} \mathrm{C}$. The residual weight of approximately $36 \%$ indicates the weight percentage of metallic impurities present in the raw MWCNTs. From the TGA pattern of

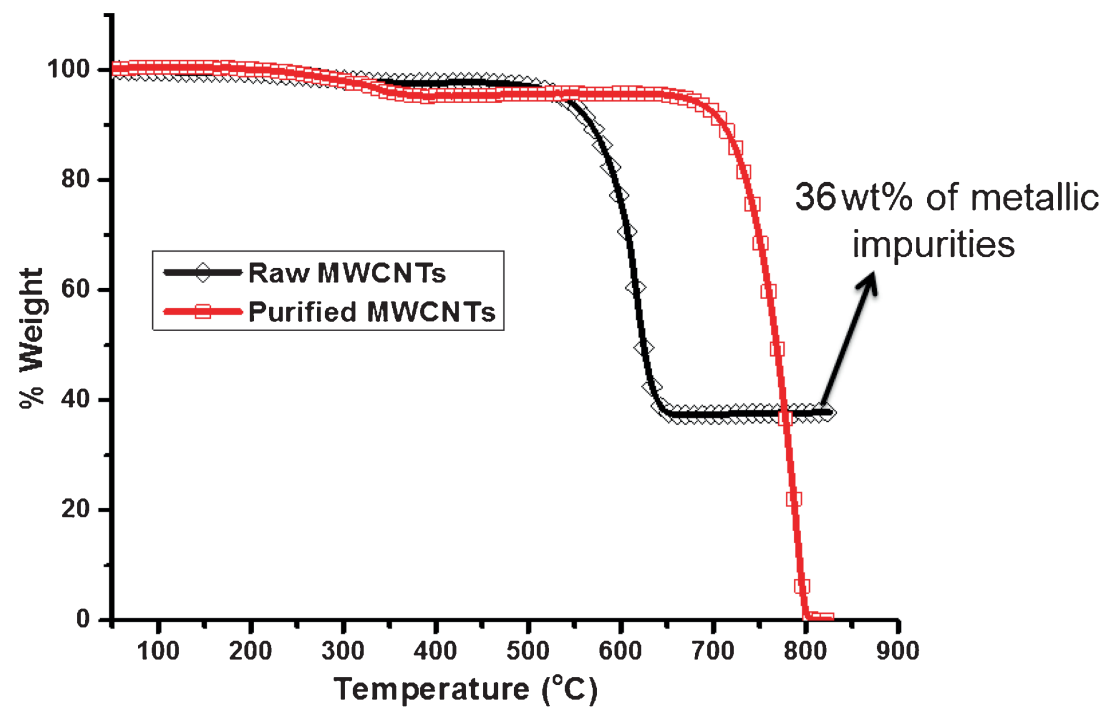

Figure 2. Graphs showing the metallic impurity level (shown by arrow) in MWCNTs as obtained from TGA studies. 


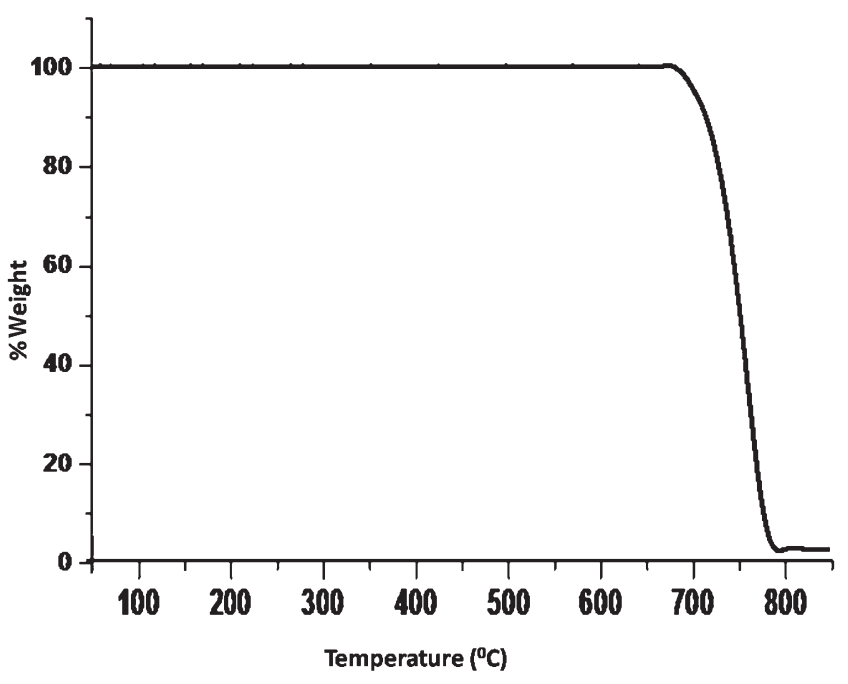

Figure 3. TGA of $120 \mathrm{~min}$ heat-treated MWCNT.

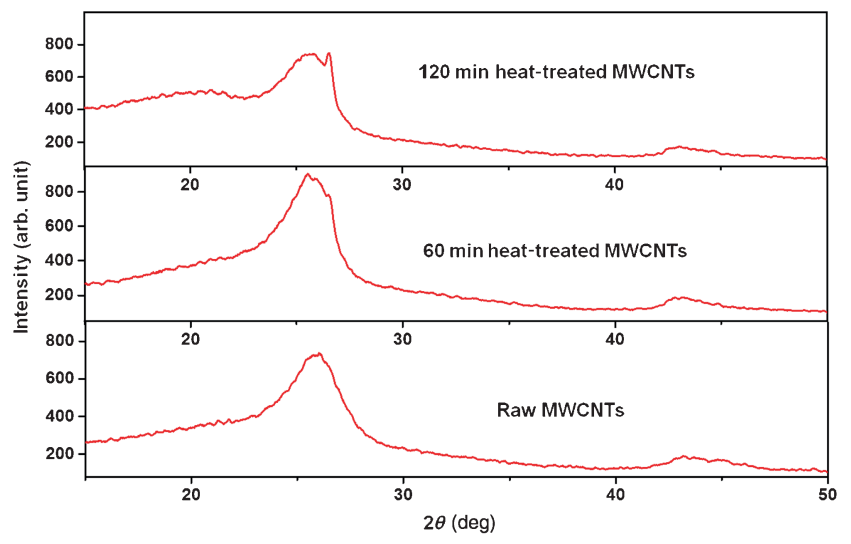

Figure 4. XRD patterns of the raw, high-temperature heat-treated MWCNTs. Peak asymmetry for 60 min heat-treated MWCNTs indicates initiation of internal distortions. Two prominent peaks for $120 \mathrm{~min}$ heat-treated MWCNTs indicate the formation of two different interlayer spacings due to structural degradation.

60 min heat-treated (purified) MWCNTs it can be seen that the residual weight drops to base value. This indicates the absence of any metallic impurities. It can be seen from the TGA curves that 60 min heat-treated MWCNTs are stable to up to $700^{\circ} \mathrm{C}$ where as raw MWCNTs were oxidized at $550^{\circ} \mathrm{C}$ itself (figure 2). This indicates enhanced thermal stability for the 60 min heat-treated MWCNTs. This could be attributed to the fact that in the absence of metallic impurities (which were removed by evaporation) there is no catalyst which can promote the oxidative decomposition of MWCNTs at lower temperatures. Besides this, high-temperature heat treatment is known to remove the microstructural defects of MWCNTs which also contributes to their enhanced thermal stability [8].

The TGA curve of the 120 min heat-treated MWCNTs is shown in figure 3 , which also has shown the trend similar to 60 min heat-treated MWCNTs in terms of purity and thermal stability of MWCNTs.
Table 2. Inter layer spacing, full-width half-maxima (FWHM) of MWCNTs.

\begin{tabular}{lccc}
\hline MWCNTs & $2 \theta_{(002)}$ & $d_{(002)}(\mathrm{nm})$ & $\operatorname{FWHM}_{(002)}\left({ }^{\circ}\right)$ \\
\hline Raw & 25.83 & 0.3445 & 1.92 \\
60 min heat treated & 25.46 & 0.3493 & 1.34 \\
& 26.56 & 0.3352 & 0.28 \\
\multirow{2}{*}{120 min heat treated } & 25.51 & 0.3469 & 1.34 \\
& 26.37 & 0.3357 & 0.28 \\
\hline
\end{tabular}

XRD patterns of raw MWCNTs, 60 min heat-treated MWCNTs and 120 min heat-treated MWCNTs are shown in figure 4. XRD peak observed near $2 \theta$ value of $26^{\circ}$ corresponds to (002) plane, while the peak that was observed near $2 \theta$ value of $42^{\circ}$ corresponds to (100) plane (figure 4). Position and the intensity of the (002) peak indicate the interplanar spacing while the (100) plane indicates the in plane graphitic structure [8]. Ideal graphite has got an interlayer spacing of $0.3348 \mathrm{~nm}$, while the turbostatic graphite shows an interlayer spacing of $0.344 \mathrm{~nm}$. Smaller (002) interlayer spacing, smaller full-width half-maxima (FWHM) of (002) and higher (002) intensities indicate a highly developed graphitic structure. By using Bragg's law interlayer spacing was calculated for raw, 60 min heat-treated and $120 \mathrm{~min}$ heat-treated MWCNTs which are shown in table 2.

It can be seen that the interlayer spacing of the raw MWCNTs is much deviated from the ideal graphitic structure. This is because raw MWCNTs are generally synthesized through CVD method, where the synthesis temperatures are generally in the range of $1000^{\circ} \mathrm{C}$. As the MWCNTs were not exposed to graphitization temperatures during synthesis, they would not show ideal graphitic structure. Besides this, during the formation of MWCNTs in CVD chamber, different precipitation rates of the carbon atoms through catalyst are possible which give a high degree of disorder (undulated fringes). This could result in more deviation in interlayer spacing as compared with ideal graphitic structure for the raw MWCNTs [13].

TEM images of MWCNTs are shown in figure 5. Raw MWCNTs have shown metallic impurities (figure 5a). During high-temperature heat treatment, metal impurities were eliminated by evaporation, while simultaneously structural perfection has increased. This can be evidenced from the TEM images of the 60 min heat-treated MWCNTs, which have shown linear, stiff graphene layers along the tube axis (figure $5 \mathrm{~b}$ ). This is because high-temperature heat treatment can give required thermal energy for the diffusion of the atoms, and thus reorient the structure close to the ideal graphitic structure [13]. The interlayer spacing values obtained from the XRD (table 2) for the 60 min heat-treated MWCNTs $(0.3352 \mathrm{~nm})$ also show near ideal graphitic structure $(0.3348 \mathrm{~nm})$. This indicates that even if the MWCNTs were grown with turbostatic structure at low temperatures, near graphitic structure can be obtained with hightemperature heat treatment [16]. Slight difference between the interlayer spacing of the 60 min heat-treated MWCNTs 

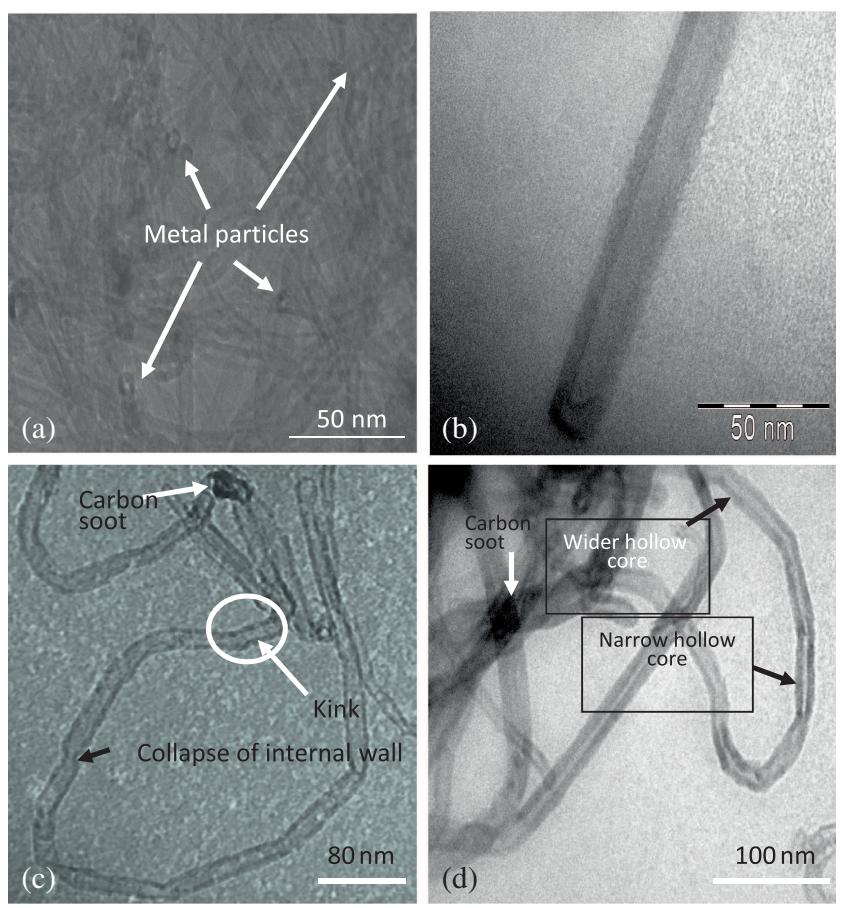

Figure 5. TEM of MWCNTs: (a) raw MWCNTs with metal impurities, (b) 60 min heat-treated MWCNTs with stiff graphene planes, (c) 120 min heat-treated MWCNTs with kinks, collapsed internal walls, deposited carbon soot and (d) 120 min heat-treated MWCNTs with varying internal hallow core diameters.

as compared with the ideal graphite interlayer spacing could be due to the restriction in the graphene sheet spacing inherent to the curved sheet form present in the MWCNTs.

XRD peak of the 60 min heat-treated MWCNTs has shown slight asymmetry near $2 \theta$ value of $26^{\circ}$, indicating initiation of degradation of MWCNTs. This asymmetry has grown into two prominent peaks for 120 min heat-treated MWCNTs, indicating that $d$-spacing of internal layers varied significantly due to structural degradation with the collapse of internal walls (figure 5c).

From Bragg's law it can be seen that there were some zones with interlayer spacing of $0.349 \mathrm{~nm}$ which is close to turbostatic graphite while the other zones were still having the interlayer spacing that is close to ideal graphite. Thus, 120 min heat treatment resulted in different interlayer spacings across the diameter of the MWCNTs. Different interlayer spacings across the diameter of the MWCNTs were also previously reported by some other research groups [6]. This can be attributed to the fact that during prolonged annealing, different layers having differing diameters experience varying degrees of internal stresses. The innermost layers which are having lowest diameter, will have more stress and will collapse ahead of other inner layers during their continuous shrinkage due to thermal annealing [11]. On the other hand, outermost layers may not experience significant stresses and thus can retain near graphitic structure. Innermost layers which collapse due to thermally induced stresses

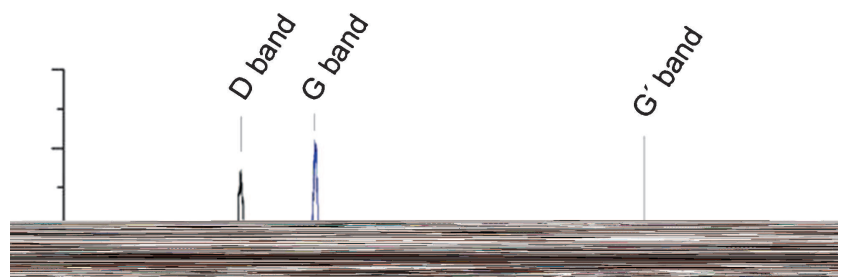

Figure 6. Raman spectra of MWCNT samples: (a) pristine MWCNT, (b) 60 min heat-treated MWCNTs and (c) $120 \mathrm{~min}$ heat-treated MWCNTs.

generate carbon species. These carbonaceous materials diffuse out and form a carbon coating on the surface of the MWCNTs (figure 5c). Enhanced diameter of the hollow core of $120 \mathrm{~min}$ heat-treated MWCNTs reinforce this mechanism (figure 5d) [13]. As the collapse of the internal layers continues, crack-like voids and kinks can develop along the tube axis (figure $5 c$ ).

Limited degradation of the innermost layers of the MWCNTs when heat treated for $60 \mathrm{~min}$ could be because of the fact that metal catalyst that is present in the raw MWCNTs may be acting as a moderating source in preventing the collapse of the inner layers. As and when carbon atoms break away from the ordered structure, the metal catalyst forms the metal carbide and again precipitates out the carbon atoms in ordered graphitic structure mimicking the CNTs growth mechanism. This kind of second growth of CNTs during high temperature annealing was reported earlier by Kim et al [13]. However, once the metal particles evaporate completely during prolonged heating, no such reverse integration of the carbon atoms that are diffusing out of the graphitic structure is possible which leads to complete collapse of the innermost walls. This mechanism is reinforced by the observation that when the heat treatment was continued for 120 min, MWCNTs with significant amount of carbon soot deposited on their surface can be seen indicating degradation of MWCNTs under prolonged heat treatment process (figure 5c). Thus it is inferred that high-temperature heat treatment for limited duration (60 min in the present study) is helping in improving the graphitic nature of the MWCNTs while prolonged (120 min in the present study) heating is resulting in significant structural distortions.

The above structural changes because of high-temperature heat treatment were also confirmed by Raman spectroscopy 
Table 3. Raman data for raw and heat-treated MWCNTs.

\begin{tabular}{lccccc}
\hline Sample & $\begin{array}{c}\text { D band } \\
\left(\mathrm{cm}^{-1}\right)\end{array}$ & $\begin{array}{c}\mathrm{G} \text { band } \\
\left(\mathrm{cm}^{-1}\right)\end{array}$ & $\begin{array}{c}\mathrm{G}^{\prime} \text { band } \\
\left(\mathrm{cm}^{-1}\right)\end{array}$ & $I_{\mathrm{D}} / I_{\mathrm{G}}(R)$ & $\begin{array}{c}\text { FWHM of G band } \\
\left(\mathrm{cm}^{-1}\right)\end{array}$ \\
\hline Raw MWCNT & 1324 & 1579 & 2643 & 1.775 & 64.6 \\
60 min heat-treated MWCNT & 1321 & 1564 & 2633 & 0.692 & 35.9 \\
120 min heat-treated MWCNT & 1321 & 1568 & 2633 & 0.793 & 28.7 \\
\hline
\end{tabular}

studies. The D band of the Raman spectrum arises out of defects present in the MWCNTs while the G band arises from the graphitic planes of the MWCNTs. Ratio of the intensities of the $\mathrm{D}$ band $\left(I_{\mathrm{D}}\right)$ to $\mathrm{G}$ band $\left(I_{\mathrm{G}}\right)$, i.e., $I_{\mathrm{D}} / I_{\mathrm{G}}(R$ value) is a reasonable index to evaluate the crystal planar domain size of graphite. Lower the $R$ value, better will be the structural perfection of the MWCNTs. Raman spectra show an increased intensity for $\mathrm{G}$ band and reduction in the intensity of the D band with $60 \mathrm{~min}$ treatment as shown in figure 6 and table 3 . This indicates that during hightemperature heat treatment, initially up to certain duration (approximately $60 \mathrm{~min}$ in the present study) defects are healed and the size of in-plane graphitic structure of MWCNTs is becoming larger. This observation is in consistence with the XRD and TEM studies. However, when the high-temperature heat treatment was continued for longer durations (120 min in the present study), severe structural degradation of the MWCNTs was observed as inferred from the $R$ value which started to increase (table 3 ).

\section{Conclusions}

High-temperature heat treatment at $2600^{\circ} \mathrm{C}$ in inert atmosphere for controlled durations (60 $\mathrm{min}$ in the present study) can give ultra-high pure MWCNTs. Enhanced structural perfection for MWCNTs can also be obtained with this method provided the high-temperature exposure of MWCNTs is restricted to controlled durations. Metal impurities which are present in the MWCNTs act as a moderating source in arresting the structural collapse of MWCNTs. Continued heat treatment of MWCNTs, even after all metal impurities are eliminated, causes structural damage to MWCNTs by collapse of internal walls and interlayer distortions of graphitic layers.

\section{References}

[1] Endo M, Saito R, Dresselhaus M S and Dresselhaus G 1997 Carbon nanotubes: preparation and properties Ebbesen $\mathrm{T} \mathrm{W}$ (ed) (USA: CRC Press) p 35

[2] Peng X H, Liu C and Cheng H M 2008 Carbon 462003

[3] Martinez M T, Callejas M A, Benito A M, Cochet M, Seeger T and Anson A 2003 Carbon 412247

[4] Xing Y, Li L, Chusuei CC and Hull R V 2005 Langmuir 21 4185

[5] Sato Y, Ogawa T, Motomiya K, Shinoda K, Jeyadevan B and Tohji K 2001 J. Phys. Chem. B 1053387

[6] Katayama T, Araki H and Yoshino K 2002 J. Appl. Phys. 91 6675

[7] Huang W, Wang Y, Luo G and Wei F 2003 Carbon 412585

[8] Andrews R, Jacques D, Qian D and Dicky E C 2001 Carbon 391681

[9] Zhang H, Sun C H, Li F, Li H X and Cheng H M 2006 J. Phys. Chem. B 1109477

[10] Wang Y, Wu J and Wei F 2003 Carbon 412939

[11] Koshio A, Yudasaka M and Ijima S 2007 J. Phys. Chem. C 11110

[12] Yadusaka M, Ichihashi T, Kasuya D, Katura H and Ijima S 2003 Carbon 411273

[13] Kim Y A, Hayashi T, Osawa K, Dresselhasu M S and Endo M 2003 Chem. Phys. Lett. 380319

[14] Kim Y A, Muramatsu H, Hayashi T, Endo M, Terrones M and Dresselhaus M S 2004 Chem. Phys. Lett. 39887

[15] Metenier K, Bonnamy S, Beguin F, Journet C, Bermier P, Lamy de la Chapelle M, Chauvet O and Lefrant S 2002 Carbon 401765

[16] Endo M, Nishimura K, Kim Y A, Hakamada K, Matushita T, Dresselhaus M S and Dresselhaus G 1999 J. Mater. Res. 14 4474 